\title{
Retour sur la genèse, l'évolution et la préservation des villes et villages miniers de Roumanie
}

About birth, development, evolution and conservation of mining towns and villages in Romania

\section{Gabriela Pașcu}

\section{OpenEdition}

\section{Journals}

Édition électronique

URL : http://journals.openedition.org/rge/9267

DOI : $10.4000 /$ rge.9267

ISSN : 2108-6478

Éditeur

Association des géographes de l'Est

\section{Référence électronique}

Gabriela Pașcu, «Retour sur la genèse, l'évolution et la préservation des villes et villages miniers de Roumanie ", Revue Géographique de l'Est [En ligne], vol. 58/3-4 | 2018, mis en ligne le 13 juillet 2020, consulté le 08 septembre 2020. URL : http://journals.openedition.org/rge/9267 ; DOI : https://doi.org/ $10.4000 /$ rge.9267

Ce document a été généré automatiquement le 8 septembre 2020

Tous droits réservés 


\title{
Retour sur la genèse, l'évolution et la préservation des villes et villages miniers de Roumanie
}

\author{
About birth, development, evolution and conservation of mining towns and \\ villages in Romania
}

Gabriela Pașcu

\begin{abstract}
«Les petites villes de Roumanie, au cours de 25 dernières années, se sont transformées en épaves. Construites en grande partie autour d'une entreprise, la faillite de cette dernière a provoqué leur agonie par le départ des jeunes vers d'autres villes ou pays, le vieillissement de la population et la réduction drastique de l'activité économique. Il s'ajoute à tout cela d'autres fardeaux comme un budget local réduit et donc des chances presque nulles qu'un investisseur s'intéressent à elles. La situation pourrait être corrigée (...), en prenant quelques décisions locales, allant de l'amélioration de l'infrastructure à l'adaptation des écoles aux nouvelles exigences (...). » (Tătar, 2016).
\end{abstract}

1 Cette citation résume bien l'état des petites villes de Roumanie et pointe le fait que beaucoup de ces petites villes sont nées et se sont développées autour de la mine et de l'industrie. En effet, la Roumanie, Pays d'Europe Centrale et Orientale (PECO), connait depuis les temps anciens un développement minier important. Ce développement a été continu, mais est longtemps resté artisanal. Le premier épisode de croissance coordonnée et industrielle de l'exploitation a finalement lieu tard, au XIXème siècle, en Transylvanie, à l'époque partie de l'Empire austro-hongrois ${ }^{1}$ (Baron, 1998). L'exploitation des mines de cette région est alors facilitée par la suppression, par le gouvernement de Vienne, des barrières douanières et par l'intégration de la Transylvanie dans le système douanier unifié de l'Empire. C'est durant cette période que s'installent de grandes entreprises étrangères ${ }^{2}$ qui domineront l'économie du territoire roumain pour longtemps.

En 1918, après la Première guerre mondiale, se forme la Grande Roumanie, par l'union des quatre principautés, dont la Transylvanie. L'unification signifie, en plus, de son importance politique et sociale, le regroupement dans un seul État et donc sous une 
seule gestion politique, des nombreuses ressources du sous-sol: charbon, fer, pétrole, etc. dans un pays où " (...) nulle part ailleurs sur le globe n'a été trouvé une variation si grande de minerai, sur une surface comme celle de notre pays." (Iancoulescu, 1928). Ainsi, l'implication de l'État a été constante pendant l'Entre-deux-guerres et, par la suite, avec plus ou moins de force, selon les circonstances politiques. Parallèlement, il faut aussi ajouter la création d'une base législative très importante, destinée à soutenir le développement du secteur industriel, y compris le secteur minier. Aussi une première phase de nationalisation partielle peut alors être identifiée avec la formation de grandes entreprises minières ${ }^{3}$, ce qui a par ailleurs favorisé l'organisation de syndicats, exigeant de meilleures conditions de vie et de travail.

Après la Seconde Guerre mondiale, en Europe, la nationalisation est considérée comme un instrument de gestion essentiel de l'économie, mais avec des implications fort différentes entre l'Est et l'Ouest du continent. Contrairement à la partie occidentale, où la nationalisation est guidée par la volonté de reconstruction économique après la guerre, la zone orientale connaît une nationalisation qui devient un instrument de contrôle politico-économique de l'Union des Républiques Socialistes Soviétiques (URSS), puissance dominante à l'Est de l'Europe après la guerre. Dans ce contexte, la loi de la nationalisation de l'industrie entre en vigueur le 11 juin 1948 en Roumanie. Depuis lors, les mesures centralisatrices s'amplifient et, durant les années 1950, la présence soviétique caractérise la vie sociale, politique, économique et culturelle. L'institution progressive de contrôles forts entraîne la subordination de l'économie roumaine aux intérêts soviétiques. Cela est marqué par l'attribution de larges pouvoirs à la Commission de Contrôle Alliée (dominée par les Soviétiques) et par la création des entreprises communes roumaines et soviétiques appelées SOVROM. Dans ce contexte, le secteur minier devient un secteur stratégique et de grande importance pour les Soviétiques et notamment pour leur dispositif militaire, avec lequel ils contrôlent l'Europe Centrale et Orientale.

De toute la période socialiste, les années 1950-1960 sont considérées comme les plus positives, parce que de nombreuses mesures ont été prises pour améliorer l'exploitation minière, à la base de tout développement de l'industrie lourde: amélioration des méthodes de travail, généralisation de la mécanisation et achèvement de l'équipement minier avec des machines d'extraction, cellules de flottation, locomotives pour les mines, etc.

5 Les années 1960, marquent, en Europe de l'Ouest, le début des fermetures d'exploitations minières, en raison des coûts trop élevés de l'exploitation et de l'entretien des sites et d'une pollution de moins en moins acceptée. Par contre, en Roumanie, le processus d'industrialisation s'intensifie, dans la plupart des cas soutenu artificiellement par le contrôle des coûts et des importations des matières premières. Cependant, en raison de la réduction des profits, les investissements technologiques ne peuvent plus se poursuivre et, encore aujourd'hui, dans des entreprises minières qui fonctionnent toujours, on peut trouver des machines qui datent des années 1920-1930. Enfin, à partir des années 1960, les mineurs, en tant que représentants majeurs de la classe ouvrière, sont utilisés comme force politique de propagande et de contrôle. Cela se poursuit d'ailleurs dans les premières années après la révolution de 1989, lors des mineriade ${ }^{4}$ de 1991 et 1992 (Pașcu, 2015).

Dans les années $1990^{5}$, l'industrie minière roumaine commence lentement son déclin, en retard donc par rapport à celui de l'Europe Occidentale. Les industries sont 
devenues trop coûteuses pour être subventionnées par l'État et le processus de privatisation commence alors. Ce processus soulève jusqu'à aujourd'hui de nombreuses questions, d'autant plus que les compagnies minières restent propriété de l'État jusqu'à leur fermeture en 2005-2006. Après leur fermeture, un grand nombre d'établissements miniers et de territoires miniers, avec leurs nombreux bâtiments et installations de valeur, sont ainsi laissés à l'abandon.

Enfin, l'adhésion de la Roumanie en 2007 à l'Union Européenne apporte de nouveaux règlements et normes pour les mines de charbon qui fonctionnent encore (Vallée du Jiu). Même s'il n'y a pas alors l'implantation de nouveaux sites d'extraction, de nouvelles constructions, soutenues par des investissements extérieurs, augmentent considérablement la taille de ceux déjà existants.

Une fois ce contexte historique de l'exploitation minière en Roumanie rappelé, cet article a pour objectif de montrer comment ont évolué récemment les territoires construits par l'exploitation minière. Dans un premier temps, il s'agira de déterminer les différents types de territoires créés par l'exploitation minière, avant d'évoquer, dans un second temps, leur déclin brutal et leur situation d'abandon actuel pour interroger, enfin, leur redéveloppement intégrant ou non des éléments industrialopatrimoniaux.

\section{1 - La mine et l'usine, forces créatrices de territoires}

L'industrie en général, et la mine en particulier, favorisent, par leur attractivité liée au besoin de main d'œuvre, la création de nouvelles communautés villageoises ou urbaines. Sur le territoire roumain, de la fin du XIXème siècle au milieu du XXème siècle, cela s'opère en trois étapes, qui se chevauchent et sont modélisées sur la figure 1.

Figure 1 : Typologie des principales implantations minières roumaines

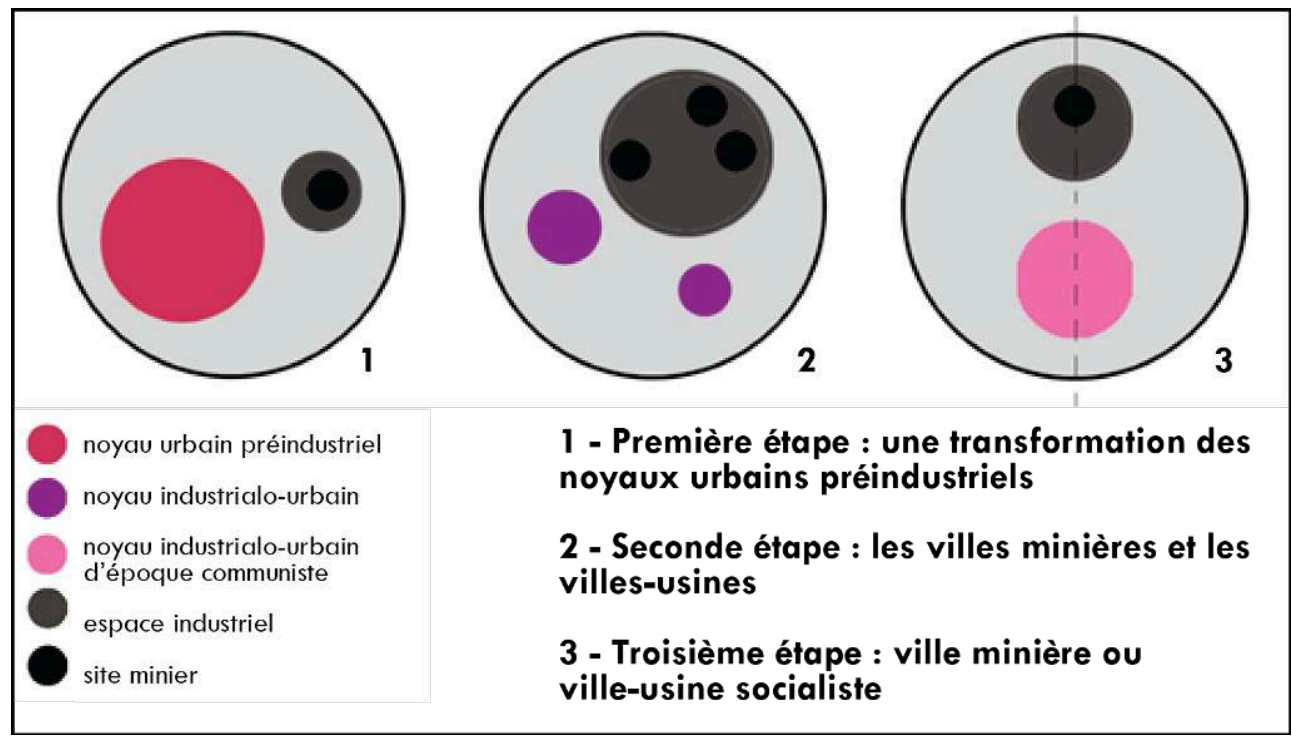

Source : Pașcu (2015)

- 1 - La première étape (de la fin du XIXème siècle jusqu'à la Première Guerre mondiale) est caractérisée par l'arrivée de spécialistes étrangers, surtout autrichiens, tchèques, italiens et français. Lors de cette étape, l'impact majeur s'est traduit par un mélange des communautés, 
qui a transformé la composition sociale de villes déjà existantes (noyau préindustriel sur la figure).

- 2 - La deuxième étape (de l'Entre-deux-guerres aux années 1970) est caractérisée par le déplacement de la main d'œuvre vers les grandes régions minières (Monts Apuseni ${ }^{6}$, Vallée du Jiu, bassin de Motru, région de Baia Mare, bassin de Comăneşti, Anina, etc.). Cette arrivée provoque la croissance des villes et des villages entourant les mines et usines, transformant plus fortement le territoire que lors de la première étape.

Les grandes compagnies minières s'impliquent dans la qualité de vie de mineurs et des travailleurs par la construction de logements et des fonctions auxiliaires, créant de nouveaux quartiers, voire de nouvelles villes (fig. 2). Ces stratégies sont comparables à celles pratiquées par les entreprises de l'Ouest de l'Europe. L'habitat le plus souvent développé a été de type pavillonnaire, se rapprochant des cités ouvrières d'Europe occidentale et appelées colonii : des maisons détachées les unes des autres, construites pour deux à quatre familles, avec accès indépendant pour chacune. Ces colonii se trouvent à proximité des sites d'exploitations ou de traitement.

Figure 2 : Anina, Puits 1 et ses colonii

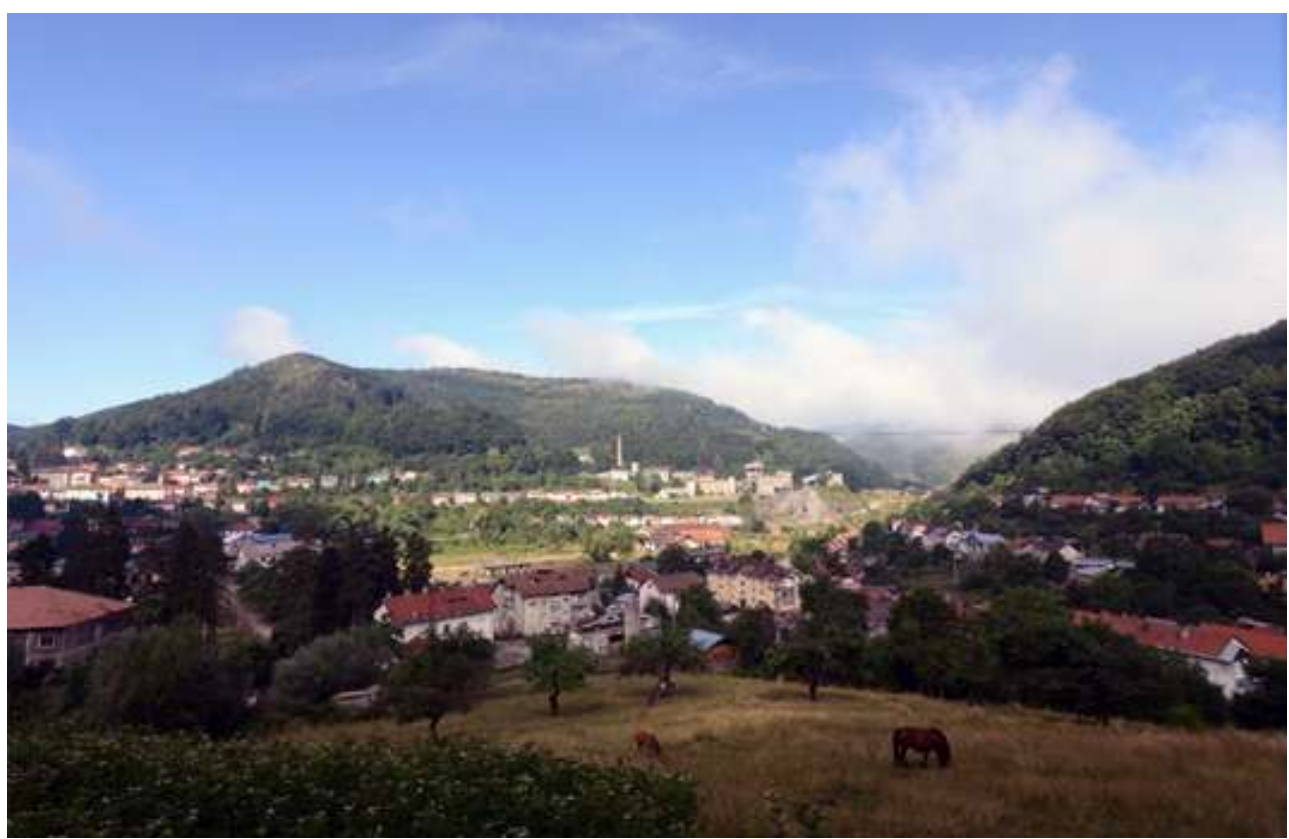

Source : Mina de Idei Anina, 2015

- 3 - Les grands ensembles, d'inspiration soviétique, forment la troisième étape. Ils commencent à se construire après les années 1950 et ces logements constituent le programme architectural le plus important associé au développement industriel, parfois soutenu par d'autres types de constructions sociales, culturelles, sanitaires et sportives (ex. : ville de Brad, village de Gurabárza, ville d'Anina, ville de Petroșani, etc.). Les formes les plus couramment utilisées sont d'influence soviétique, avec, dès la période 1952-1958/1959, la multiplication des blocs (Socialisme Réaliste) (fig. 3 et 4). Après 1959, l'influence est plus internationale avec le courant moderniste (Tulbure, 2016). "Le développement rapide de notre industrie a forcé la construction d'innombrables logements à côté des industries émergentes ou nouvellement construites pour le logement optimal des travailleurs. Pour les industries importantes, ces ensembles atteignent la taille des villages ou même des villes réelles avec une population de plusieurs milliers d'habitants. Dans la plupart des cas, ils ont le volume d'un quartier ou d'une petite ville, avec une population de 500 à 7000 habitants." (Lăzărescu, 1954). 
Figure 3 : Colonii de travailleurs, Uricani, Vallée du Jiu

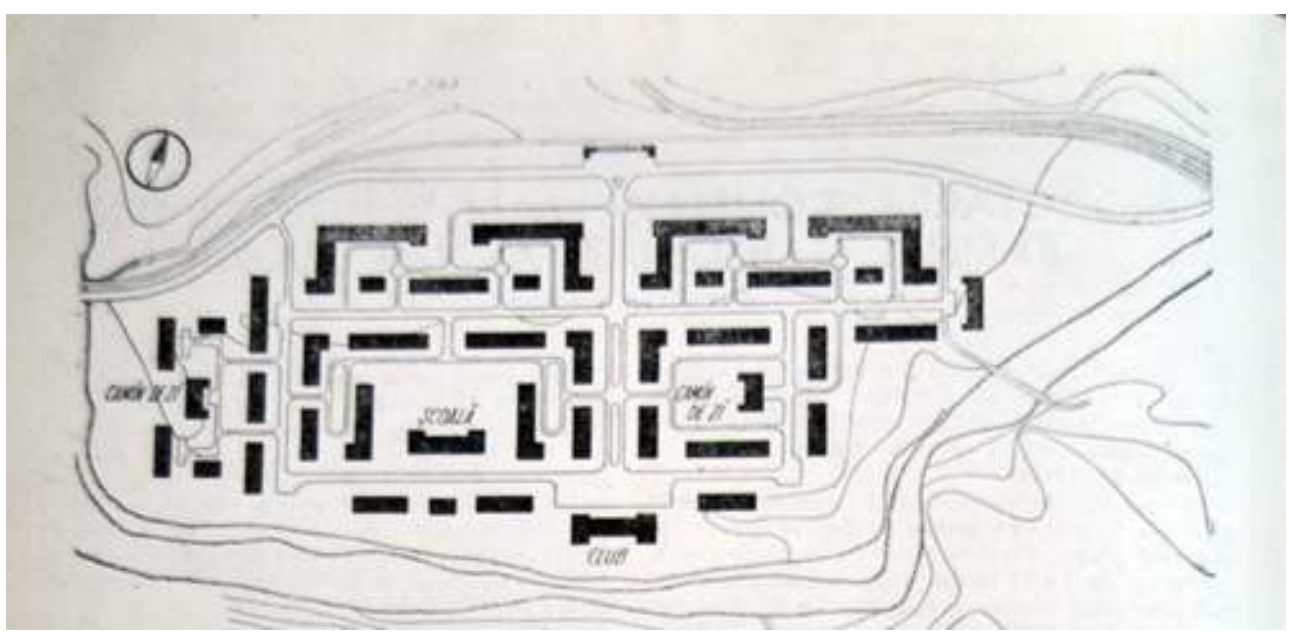

Source : Vernescu (1952)

Figure 4 : Vue du quartier ouvrier, Vulcan, Vallée du Jiu

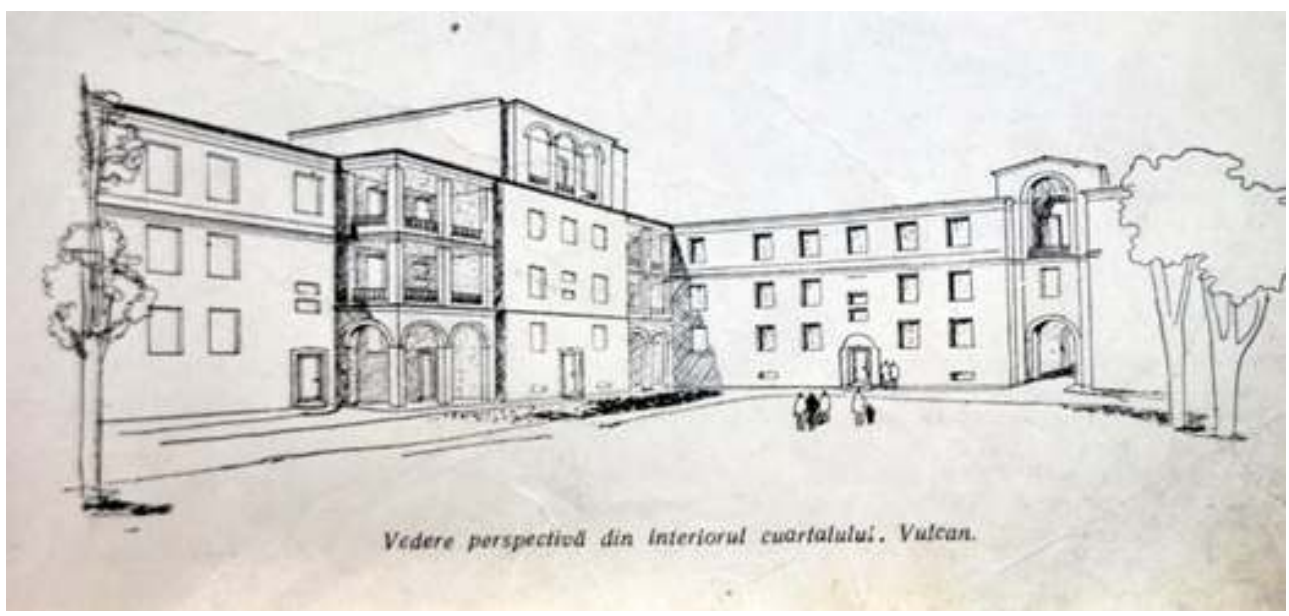

Source : Vernescu (1952)

9 L'année 1966 constitue un tournant car, désormais, la possession d'appartements privés indépendants de l'État est autorisée. La variété des formes augmente, mais la qualité des logements subventionnés par l'État diminue. L'accent est mis par le pouvoir politique sur les blocs d'appartements, avec de plus en plus d'étages; ils constituent alors le programme principal de la politique du logement ouvrier. "Le rapport entre les blocs hauts et moyens d'un côté et bas de l'autre a évolué en faveur des plus élevés : entre 1951 et 1960, 81,4\% étaient des blocs de 5 étages ou moins; entre 1960 et 1970, 76,4\% ; entre 1977 et 1980, 62\% ; entre 1981 et 1990, 50\%." (Zahariade, 2011).

Le bloc d'appartements est à cette époque la meilleure façon de coller à la politique et aux discours d'égalité entre les Hommes et à un mode de vie standardisé et contrôlé. Dans un premier temps, ces ensembles de blocs qui entourent des jardins peuvent être considérés comme une réinterprétation de l'habitat en cité-jardin. Dans un second temps, les ensembles perdent en cohérence, abandonnant l'idée de créer une ambiance, une organisation claire en rues bien structurées. De plus, dans le cas des villes minières socialistes, l'idée de "réorganiser" le centre-ville, pour démontrer la puissance 
industrielle et politique est devenue très fréquente. La réorganisation du centre-ville implique donc des actions de transformation, de doublement ou même de déplacement vers les nouveaux quartiers, qui ont souvent plus de fonctions sociales que les centres anciens. Finalement et malgré ces transformations, la majorité des villes minières roumaines conservent, dans leur empreinte urbaine, toutes ces différentes étapes architecturales, liées au développement de l'industrie minière (fig. 5).

Figure 5 : Les deux noyaux urbains de Baia de Arieș, ancienne ville minière

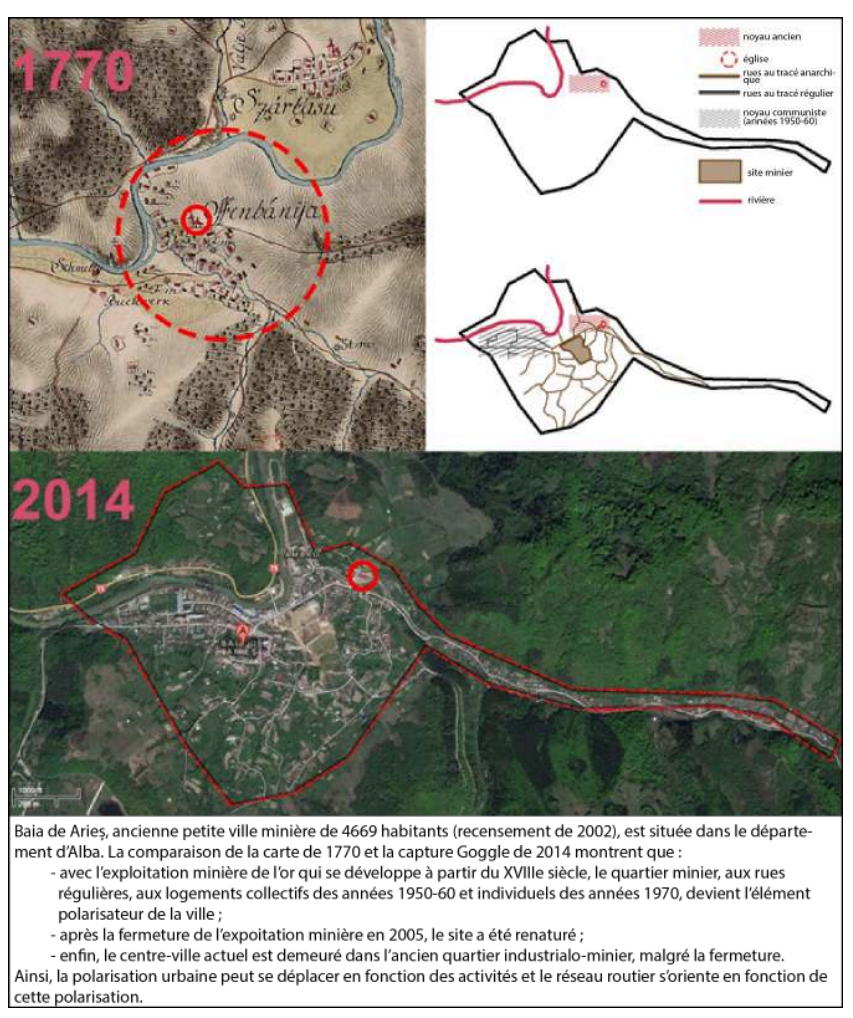

Source : G. Pașcu (2015)

\section{2 - Déclin, abandon et questions patrimoniale et d'identité territoriale dans les anciennes villes minières}

11 Presque comme partout en Europe, les villes minières roumaines basculent, à la fin du XXème siècle, dans la catégorie des villes rétrécissantes (Florentin et al., 2009 ; Fol \& Cunningham-Sabot, 2010). Elles ont ainsi dû faire face à :

- la fermeture du principal catalyseur de la vie, la mine ;

- un fort dépeuplement, avec des migrations massives vers d'autres pays de l'Union Européenne et vers les villes attractives de la Roumanie ;

- un chômage croissant, en raison de la fermeture des exploitations minières et des fonctions secondaires associées ;

- une faible qualité de vie ;

- une population vieillissante ; 
- une contraction urbaine dont la perception négative est souvent plus importante que la réalité : «(..) le rétrécissement urbain ne signifie pas seulement l'abandon voire la destruction de surfaces bâties, mais est aussi une affaire de perception et de vécu. La diminution des surfaces bâties est un problème, mais le côté imprécis et indésirable du phénomène en accentue la perception négative.» (Vais, 2015);

- de vastes surfaces anciennement industrielles, dispersées, à la recherche de nouveaux usages et portant de nombreux éléments patrimonialisables.

- une dilution des communautés en absence de l'élément fédérateur qu'était la mine.

- Il existe un débat concernant le nombre des villes moyennes à petites et de villages miniers ou mono-industriels en Roumanie. Les sources varient en effet selon deux études importantes :

- «Atlas territorial de la Roumanie» (Rey et al., 2006);

- «Les villes mono-industrielles de la Roumanie : entre industrialisation forcée et déclin» (Dumitrescu, 2008).

12 La différence entre ces deux études est significative. Dans la première étude, il existe 187 villes mono-industrielles en Roumaine ; dans la seconde, elles ne sont que 51, dont 33 villes dans l'industrie manufacturières et 18 villes dans l'industrie extractive. Cette différence de décompte complique encore la prise de décisions concernant les actions possibles de redéveloppement. Malgré cela, il est possible de classer ces villes en trois catégories, selon leur localisation :

- 1 - petites et moyennes villes sous l'influence de centres urbains régionaux ${ }^{7}$ (Ghelari, Teliucul sous l'influence de Hunedoara, etc.) ;

- 2 - petites et moyennes villes isolées (Bucium, Izvoru Ampoiului, Baia de Arieș etc.) ;

- 3 - petites et moyennes villes qui, en raison de leur grande proximité physique, auraient la chance de se développer en commun (Vallée du Jiu);

13 Ces trois situations sont extrêmement différentes et les actions qui pourraient être prises pour améliorer ces villes minières sont donc aussi différentes. La majorité des entreprises minières roumaines, quel que soit le type d'exploitation, commence à fermer en 2000, processus qui prend fin en 2006-2007. Les 20 ans qui se sont écoulés depuis le début des fermetures ont été suffisants pour que soient détruits la plupart des biens patrimoniaux et des paysages miniers roumains, et insuffisants pour comprendre et préparer des actions de réévaluation du patrimoine (urbain, architectural, paysager) par les communautés impliquées.

14 À partir de 2006, l'État roumain tente d'encourager les concentrations économiques territoriales à travers des politiques publiques, mais parfois sans connaitre la vraie situation du territoire. De nombreux outils, souvent liés à des zonages spécifiques, ont été mis au point pour redynamiser le territoire d'un point de vue général et d'un point de vue local : les parcs industriels, les parcs scientifiques et zones technologiques, les zones défavorisées, les zones de restructuration industrielle, les zones assistées, les incubateurs d'entreprises, les zones de montagne défavorisées, les zones minières défavorisées, etc.; des exemples sont placés sur la figure 6. La majorité de ces programmes est terminée, mais leur impact sur le développement économique et social n'a pas vraiment été évalué par des études spécifiques. 
Figure 6 : Exemple de zonages d'aide concernant les communes minières en Roumanie dans les 20 dernières années

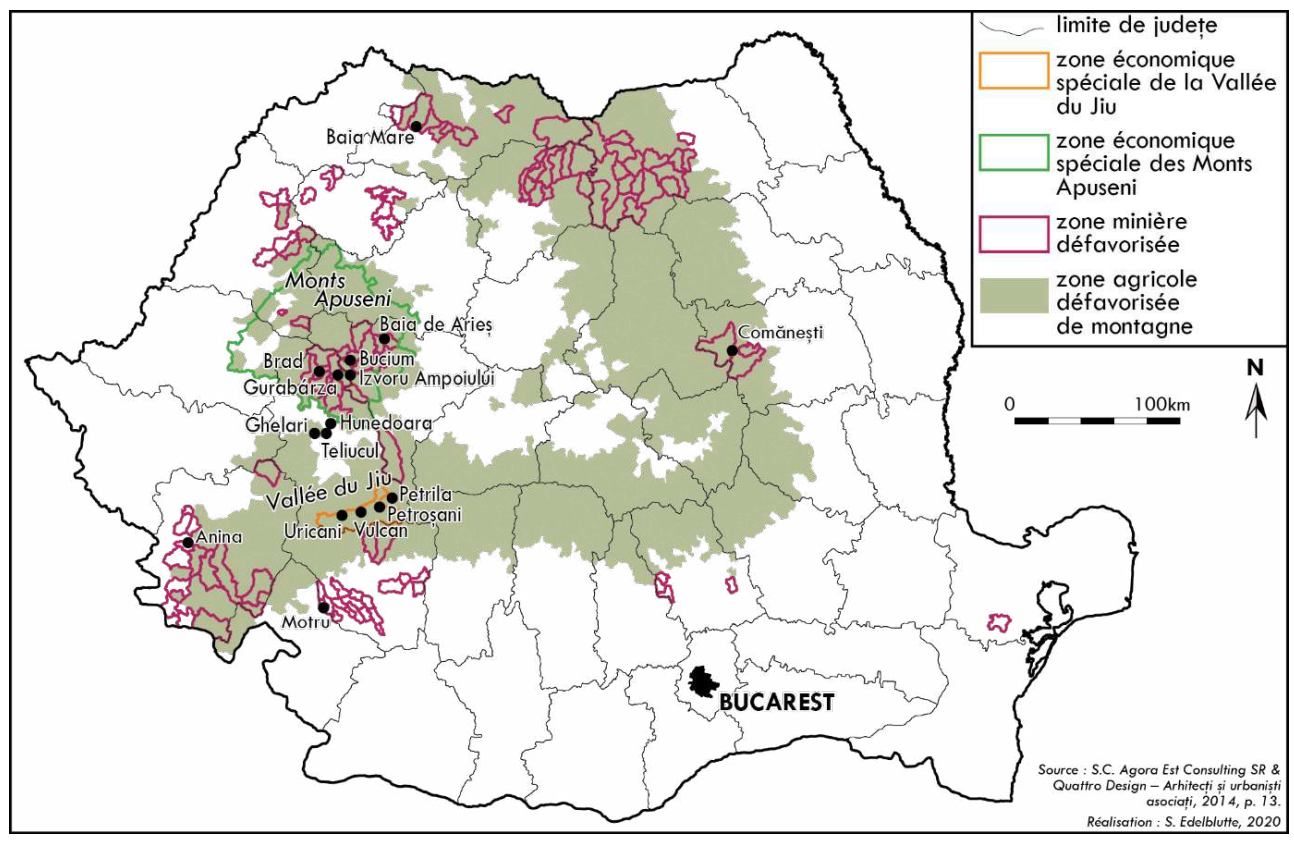

Source : S.C. Agora Est Consulting SR \& Quattro Design - Arhitecți şi urbanişti asociați, 2014, p. 13.

Tout au long de ces années de déclin et d'abandon, les anciens établissements miniers roumains sont dans une continuelle, mais peut-être inconsciente, recherche d'identité. Certaines villes minières ne conservent que des fragments de paysages ${ }^{8}$ miniers : un chevalement, quelques corons, un carreau minier, etc. Pour d'autres localités, la disparition complète des traces physiques de l'industrie et/ou de la mine fait que seul un œil exercé peut comprendre ces paysages anthropiques.

Dans ce mouvement de déclin et de déni, seules quelques villes minières s'ouvrent à la possibilité de valoriser leur patrimoine minier et/ou industriel (architecture, urbanisme, paysage, patrimoine matériel et immatériel). Parmi elles, il y a Anina (comté de Caras-Severin), Petrila (Comté de Hunedoara), Baia Sprie (comté de Maramureș), Brad (comté de Hunedoara). Ces localités entament, dans les années 2010 et jusqu'à présent, des projets culturels soutenus par des Organisations Non Gouvernementales (ONG), mais avec très peu de ressources financières. Les programmes culturels ciblent généralement la sensibilisation des populations aux héritages miniers et industriels qu'ils ont encore sur leur territoire.

Ainsi, et malgré la pauvreté des ressources, des résultats significatifs sont obtenus, par exemple :

- Certains bâtiments du site de Petrila, dans la Vallée du Jiu, ont été classés comme monuments d'intérêt national en 2015, suite à une vaste action engagée depuis 2011-2012, nommée Planeta Petrila (Planète Petrila - fig. 7). Il s'agit d'un projet de régénération urbaine initié par le dessinateur Ion Barbu, présenté par Andrei Dăscălescu dans le documentaire éponyme et mis en œuvre par divers acteurs : l'association AsociaȚia Culturală CondiȚia Română, Plusminus, Ideilagram, ACV Petroșani et Planeta Petrila. La mine de Petrila est donc sauvée de la démolition et le processus de réutilisation adaptative est lancé. L'objectif à long terme est de transformer l'ancien complexe minier en un centre économique, administratif et socio-culturel pour la région. L'approche est néanmoins affectée par la 
rigidité des procédures législatives dans le domaine de la fermeture des mines. Parallèlement, il offre des opportunités complémentaires: la promotion du patrimoine minier et ferroviaire, la présentation de la vallée du Jiu comme destination touristique intégrée, l'éducation informelle, l'inclusion sociale ou la démocratie participative.

Figure 7 : Exemples d'événements culturels à la mine de Petrila

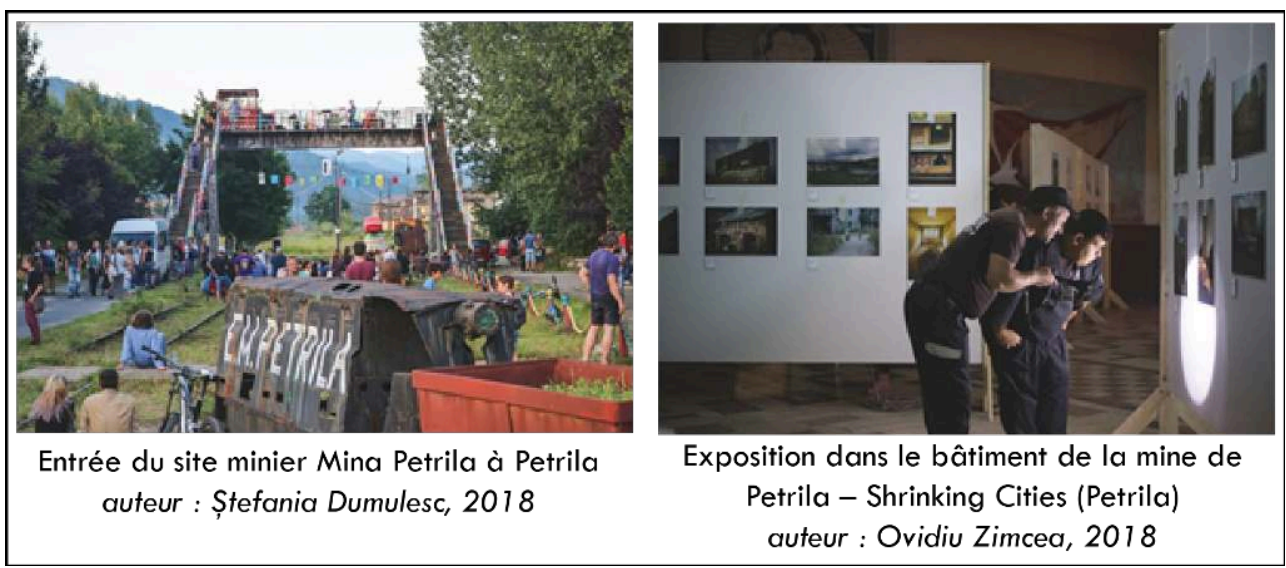

Source : http://arhitectura-1906.ro/2019/02/cum-a-devenit-petrila-o-planeta-de-la-activism-laprogram-de-regenerare-a-patrimoniului-prin-initiative-culturale/

- Des projets de reconversion sont en œuvre pour les puits d'Anina, notamment pour le puits 1. Mina de Ideai (mines des idées) Anina (2014-2019) est un projet culturel pluriannuel de mise en valeur du patrimoine industriel de la région, en impliquant la communauté locale et les spécialistes. Il est porté par l'association Alba Verde et l'association PACT (AsociaȚia pentru Patrimoniu Activ). Les résultats du projet sont nombreux : plusieurs publications ; des ateliers avec des étudiants en architecture, urbanisme et paysage ; des visites guidées et la collaboration de tous ces acteurs à l'élaboration du projet de restauration Putul I Anina (Puits I Anina - fig. 8). 
Figure 8 : vues et plan d'ensemble du Puits I à Anina

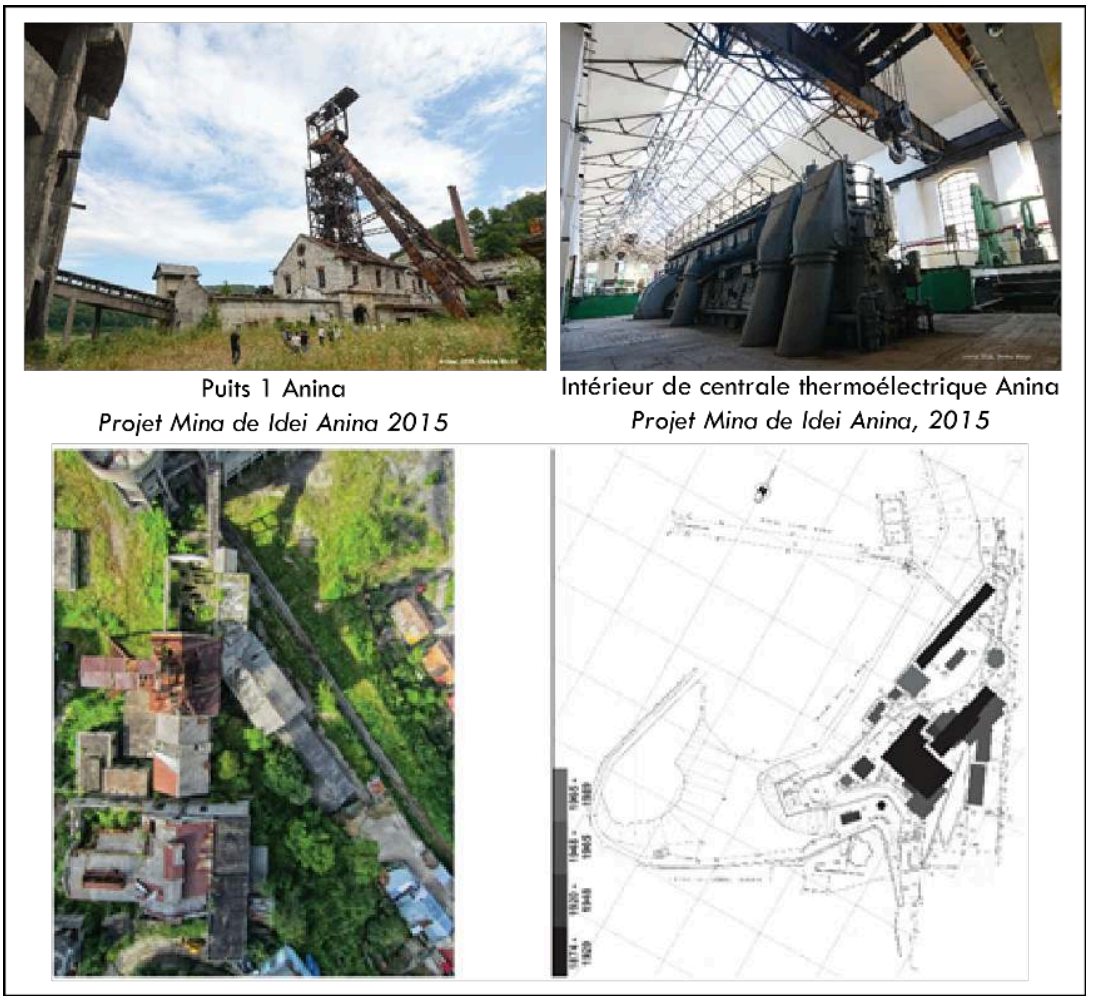

Source : Projet Mina de Idei Anina

- $\circ$ Des débats, des expositions et un atelier de création impliquant élèves locaux et étudiants d'architecture, d'urbanisme et de géographie, mais aussi des itinéraires touristiques sont proposés à Brad, Anina, Petrila et Baia Sprie. Ainsi, dans cette dernière ville, à partir de 2009, une stratégie, initiée par l'association NOD (Networking with Opportunities and Design), a été mise en place pour la création d'une aire protégée Dealul Mina (colline minière), autour d'un parc à thème (industrialo-géologique). Il s'agit de remodeler les espaces publics qui relient la colline à l'aire protégée de l'ancien centre. Les ateliers ont réuni des équipes pluridisciplinaires (architectes, paysagistes, sociologues et géologues), avec pour mission de dresser un tableau complet de la situation existante et des possibilités de revitalisation de la zone étudiée.

- Enfin, à la mine de Brad, un projet culturel de sensibilisation aux valeurs du patrimoine industriel de l'ancienne mine d'or Gurabárza (fig. 9) a été porté par l'association PACT déjà présente à Anina. Appelé Peisaje interioare. Despre mina si oameni la Brad (Paysages intérieurs: la mine et les gens de Brad), il a débouché sur 2 ateliers de travail avec les étudiants et des débats publics sur ce sujet. 
Figure 9 : Site minier d'exploitation de l'or Gurabárza près de Brad

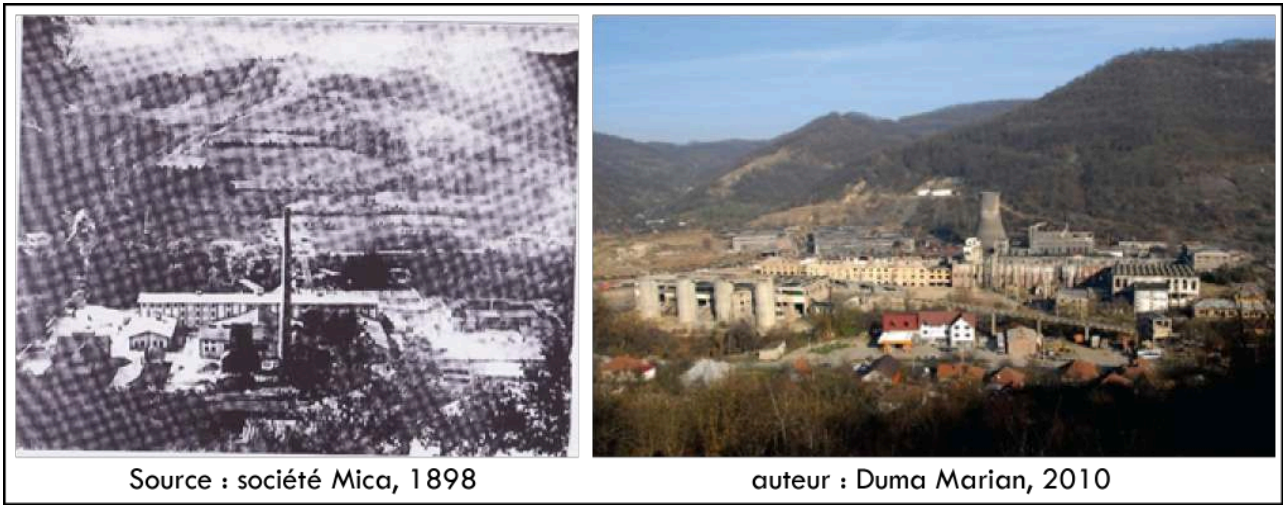

Source : projet Peisaje Interioare. Despre oameni si mina la Brad

Les actions avancent néanmoins difficilement et demandent beaucoup d'efforts, bien que le cadre juridique pour la protection, conservation et mise en valeur de ces ensembles existe en Roumanie ${ }^{9}$. Cependant, il manque encore une conscience de la valeur culturelle et de la possibilité d'utilisation du patrimoine industriel, tant au niveau de l'État que de la société civile, des médias et parfois aussi des professionnels. Il n'y a pas donc pas encore une intégration systématique de la patrimonialisation dans les anciens territoires industriels successivement réorganisés. C'est pour cela que la plupart des initiatives sont ascendantes (bottom-up), sans avoir beaucoup de soutien des gouvernements, local ou national, ou des communautés locales.

\section{Conclusion}

La Roumanie, comme tous les PECO, présente donc une histoire industrielle et minière de plusieurs siècles. Comme ses voisins, elle a connu deux principales phases d'industrialisation. La première, touchant les régions minières, s'étale de la protoindustrie au début du XXème siècle et diffère peu, si ce n'est en intensité, de ce qu'a connu l'Europe occidentale ; la seconde correspond à la période communiste de l'après seconde guerre mondiale années 1940 aux années 1980 et privilégie une industrialisation lourde et à marche forcée. L'effondrement des régimes communistes, en Roumanie comme chez ses voisins, a inauguré une intense phase de rétraction industrielle avec, en miroir, une phase de deuil (Grossetti et al., 1998 ; Daviet, 2006) particulièrement profonde et longue. Elle ne semble d'ailleurs pas terminée puisque les abandons, destructions et non-considérations des héritages miniers et industriels comme un patrimoine digne d'intérêt, sont encore largement dominants. Toutefois, quelques exemples évoqués dans le texte montrent une prise de conscience réelle, bien qu'encore légère et plus tardive, peut-être pour des raisons financières et de niveau de développement, que dans d'autres PECO (Pologne, République Tchèque).

Le but de cet article a été de présenter l'évolution et l'état actuel des villes et villages miniers des régions roumaines. Aux niveaux européen et international, ils sont encore peu connus, malgré un nombre important d'anciens établissements et leur vaste superficie, résultats de la longe et diverse histoire minière et industrielle, notamment 
autour de l'or et du charbon. Cet héritage façonne la croissance actuelle des villesusines ou villes minières de 3 façons principales :

- 1 - Les sites industriels sont devenus des sources de spéculation immobilière (dans le cas des villes moyennes et grandes) et leur utilisation est radicalement différente de l'originelle.

- 2 - Ils ont souvent été laissé à l'abandon.

- 3 - Un petit nombre seulement de sites et de bâtiments ont été reconvertis sans destruction. En effet, contrairement à d'autres pays de la zone occidentale et centrale, la régénération des anciennes zones miniers par le biais de projets appliqués n'a pas été considérée comme d'importance nationale.

21 La situation des villes et villages miniers est très bien expliquée par P. Wirth en 2012 : “(...) ces régions reçoivent un financement spécial et font face à une forte concurrence d'autres régions moins développées pour le soutien des fonds européens. Les régions dominées par une réseau urbain de petites et moyennes villes, en particulier, doivent rivaliser pour obtenir des financements et mobiliser des ressources déjà limitées".

Malgré tous ces problèmes, les initiatives plus ou moins avancées évoquées ci-dessus, devraient contribuer à changer la perception de ces régions et sites; mais ce qui manque toujours, ce sont des études (inventaires, analyses) pour bien comprendre les anciens territoires miniers et industriels ainsi que des projets de mise en valeur ou de réutilisations. Si ce manque ne peut pas être généralisé au niveau de chaque région, ville, bâtiment ou site partout en Roumanie, il faut espérer que, avec la menace de disparition imminente de ces héritages, des projets plus nombreux commenceront à voir le jour.

\section{BIBLIOGRAPHIE}

- Baron M., 1998, Cărbune și societate în Valea Jiului - perioada interbelică, Petroșani, Editura Universitas, $63 \mathrm{p}$.

- Daviet S., 2006, L'évolution du concept de reconversion : de la substitution des activités au redéveloppement des territoires. In : Daumalin X., Daviet S. \& Mioche Ph. - dir., Territoires européens du charbon, des origines aux reconversions, Aix-en-Provence, Publications de l'Université de Provence, p. 243-255.

- Dumitrescu B., 2008, Orașele monoindustriale din România: între industrializare forȚată și declin, București, Editura Universitară, 94 p.

- Edelblutte S., Paysages et territorires de l'industrie en Europe. Héritages et renouveaux. Edition Ellipses, 2009.

- Florentin D., Fol S. \& Roth H., 2009, « La "Stadtschrumpfung” ou "rétrécissement urbain" en Allemagne : un champ de recherche émergent », Cybergeo : European Journal of Geography, https://journals.openedition.org/cybergeo/22123, consulté le 19/12/2017.

- Fol S. \& Cunningham-Sabot É., 2010, « Déclin urbain » et Shrinking Cities : une évaluation critique des approches de la décroissance urbaine, Annales de Géographie, n 674, p. 359-383. 
- Grossetti M., Beslay C., Salles D., Guillaume R., Daynac M. \& Tautelle F., 1998, La construction des politiques locales. Reconversions industrielles et systèmes locaux d'action publique, Paris,

L'Harmattan, 224 p.

- Iancoulescu A. P., 1928, Les richesses minières de la nouvelle Roumanie, Thèse de doctorat, Paris, Librairie Universitaire J. Gamber, 348 p.

- Lăzărescu C., 1954, În legătură cu proiectarea așezărilor muncitorești (En relation avec la conception des établissements ouvriers), Arhitectura RPR, nr.8, p. 1.

- Pașcu G., 2015, Le patrimoine industriel - minier facteur de développement territorial. Complexité et enjeux en Roumanie, en comparaison avec la France et la Grande-Bretagne, Timișoara, Editura Politehnica, 97 p.

- Poussou J.-P., Lottin A., Naissance et développement des villes minières en Europe, Artois Presses Université Collection Histoire, 2004.

- Revue arhitectura - 1906.

- Rey V., Groza O., Ianoş I. \& Pătroescu M., 2006, Atlasul României, Enciclopedia RAO, Bucureşti (publié en français chez La Documentation française en 2007).

- S.C. Agora Est Consulting SR \& Quattro Design - Arhitecţi şi urbaniști asociaŢi, 2014, Strategia de dezvoltare teritorială a României. Studii de fundamentare (Stratégie de développement territorial de la Roumanie. Etudes fondamentales), Raport de sinteză, Ministerul Dezvoltării Regionale şi AdministraŢiei Publice, 37 p., http://sdtr.ro/upload/STUDII/ 23.\%20Sinteza_Zone\%20cu\%20specific\%20geografic_.pdf consulté le 18/12/2017

- Tătar F., 2016, Orașe mici din România proceduri de resuscitare, SINTEZA - Revista de cultură și gândire strategică, http://revistasinteza.ro/orase-mici-din-romania-proceduri-de-resuscitare/ consulté le 20/12/2017.

- Țiganea O. \& Pașcu G., 2017, Peisajul industrial Anina : Reprezentări și interpretări patrimoniale, Alba Iulia, $121 \mathrm{p}$.

- Tulbure I., 2016, Arhitectură și urbanism în România anilor 1944-1960: constrângere și experiment, Editions Simetria, București, $157 \mathrm{p}$.

- Vais D., 2015, Contracția urbană. Contracția fizică, catalogue de l'exposition Shrinking Cities Roumanie, București.

- Vernescu D., 1952, Proiectarea complexă a locuinȚelor din basinul carbonifer al Văi jiului (Conception complexe des habitations du bassin carbonifère de la Vallée du Jiu), Arhitectura RPR, nr. 6,7, p. 9 .

- Wirth P., Harfst J., Challenges of post-mining regions in Central Europe. https://www2.ioer.de/ recherche/pdf/2012_wirth_challenges_of_post-mining_regions.pdf

- Zahariade A. M., 2011, Arhitectura în proiectul comunist. România 1944 - 1989., Editions Simetria, București, 49 p.

\section{Ressources en ligne sur les sites miniers mentionnés :}

- Green Recreation / N.O.D, Baia Sprie (2009-2014) : http://tabaradecreatie.blogspot.com/

- Projet Anina, projet Mina de Idei Anina/ Anina Mines d'Idées :

https://issuu.com/asociatiaalbaverde/docs/00_mina_de_idei_anina 2017 
https://issuu.com/asociatiaalbaverde/docs/mina_de_idei_anina_-_vizita_in_comu

\section{NOTES}

1. La Transylvanie, aujourd'hui roumaine et à l'époque austro-hongroise, est une des composantes de la Roumanie, formée à la base par quatre principautés, appelées dans la langue populaire aux XIX ${ }^{\text {ème }}$ et début $\mathrm{XX}^{\text {ème }}$ siècles «les pays des Roumains »: Transilvania (la Transylvanie), Moldova (la Moldavie), Țara Românească (la Valachie) et Dobrogea (la Dobroudja).

2. Ces entreprises étaient principalement tchèques, autrichiennes (alors deux parties de l'Empire austro-hongrois), françaises et italiennes.

3. Une des plus grandes entreprises minières roumaines a été MICA - Societatea Anonimă Rom ână Minieră (Société Minière Anonyme Roumaine), fonctionnelle entre 1920 et 1948. Elle était majoritairement composée de capitaux roumains et constituait la plus grande entreprise d'exploitation minière de métaux non ferreux d'Europe Centrale et Orientale.

4. Les mineriade sont les actions de mineurs qui, entre 1991 et 1992, se sont déplacés à Bucarest pour soutenir le pouvoir politique de Ion Iliescu (alors président de la Roumanie). Ces actions ont été accompagnées par des violences.

5. L'année 1989 a été une année de changement dans le contexte politique roumain avec la transition d'un système politique communiste vers un système démocratique et capitaliste.

6. Tous les toponymes cités dans le texte sont situés sur la figure 6 .

7. Villes de taille moyenne (jusqu'à 100000 habitants).

8. Fragment de paysage : il s'agit d'une notion proposée au débat à travers le livre : «Peisajul industrial Anina: Reprezentari și interpretari patrimoniale» (Țiganea \& Pașcu, 2017). Cette notion propose une réinterprétation du concept de paysage industriel, alors considéré comme une mosaïque, composée de fragments de paysage orientés, organisés par un élément ou plusieurs éléments reliés entre eux, ici la mine ou l'industrie. Ces fragments de paysage sont aussi soumis au jeu de la perception, à la fois par les habitants de la ville et par les visiteurs extérieurs.

9. Legea nr.6/2008 - Lege privind regimul juridic al patrimoniului tehnic și industrial (Loi $n{ }^{\circ}$ 6/2008 - Loi sur le régime juridique du patrimoine technique et industriel).

\section{RÉSUMÉS}

La révolution industrielle, dans toute l'Europe, a entrainé le développement de villes et de villages entiers, a changé des destins et a accentué la multi-culturalité des territoires. L'origine de ces activités minières et industrielles est plus ou moins lointaine, mais toutes ont été confrontées à un déclin récent, voire à une disparition totale et brutale, notamment en Europe centrale et orientale. En effet, dans une Europe en reconfiguration d'un point de vue énergétique et écologique, mais aussi géopolitique, ce type d'exploitation ne peut se maintenir sans une concentration importante de force de travail et des investissements massifs dans la technologie et les infrastructures. Tout cela ne peut être mis en œuvre que dans un contexte économique favorable. 
En Roumanie, les villes et villages industriels et miniers créés par les entreprises, puis par le pouvoir communiste sont d'excellents témoignages des interactions entre l'économie, le social et le politique. Construits rapidement, ces villes et villages sont principalement le résultat d'une planification systématique, en réponse à des objectifs de rendement industriel et comme meilleur vecteur d'assurer l'hégémonie de la classe dirigeante. Cet article désire ainsi faite le point sur la genèse de ces villes et villages-usines/mines en Roumanie et sur leurs situations actuelles.

All over Europe, the industrial revolution have led to the development of cities and towns, changed destinies and accentuated the multicultural nature of the territories. The origins of mining and industrial activities are more or less distant, but all of them have faced a recent decline, or even a complete disappearance, especially in Central and Eastern Europe. Indeed, in a Europe in reconfiguration from an energetically, ecological and a geopolitical point of view, this type of exploitation cannot be maintained without a significant concentration of labor force and massive investments in technology and infrastructure. All this can only be implemented in a favorable economic context.

In Romania, the industrial mining towns and villages created by companies and the communist power, are excellent testimonies of the interaction between economy, social and political power. Built quickly, these towns and villages are mainly the result of systematic planning, in response to industrial performance objectives. This article wishes to point out the genesis of these mining/ factory-towns and mining/factory-villages in Romania and their current situations.

\section{INDEX}

Mots-clés : patrimoine industriel, Roumanie, villes et villages miniers, évolution industrielle, changements urbains

Keywords : industrial heritage, Romania, mining towns and villages, industrial evolution, urban changes

\section{AUTEUR}

\section{GABRIELA PAȘCU}

Architecte, Faculté d'Architecture et d'Urbanisme, Université Politehnica Timișoara, Roumanie e-mail : pascuggabi.arch@gmail.com 